\title{
How Flow Injection Analysis (FIA) over the past 25 years has changed our way of performing chemical analyses
}

\author{
Hansen, Elo Harald; Miró, Manuel
}

Published in:

Trends in Analytical Chemistry

Link to article, DOI:

10.1016/j.trac.2006.07.010

Publication date:

2007

Link back to DTU Orbit

Citation $(A P A)$ :

Hansen, E. H., \& Miró, M. (2007). How Flow Injection Analysis (FIA) over the past 25 years has changed our way of performing chemical analyses. Trends in Analytical Chemistry, 26(1), 18-26.

https://doi.org/10.1016/j.trac.2006.07.010

\section{General rights}

Copyright and moral rights for the publications made accessible in the public portal are retained by the authors and/or other copyright owners and it is a condition of accessing publications that users recognise and abide by the legal requirements associated with these rights.

- Users may download and print one copy of any publication from the public portal for the purpose of private study or research.

- You may not further distribute the material or use it for any profit-making activity or commercial gain

- You may freely distribute the URL identifying the publication in the public portal

If you believe that this document breaches copyright please contact us providing details, and we will remove access to the work immediately and investigate your claim 


\title{
How Flow Injection Analysis (FIA) over the past 25 years has changed our way of performing chemical analyses
}

\author{
Elo Harald Hansen ${ }^{\mathrm{a}^{* 1}}$ and Manuel Miró ${ }^{\mathrm{b}}$ \\ a Department of Chemistry, Technical University of Denmark, Kemitorvet, Building 207, \\ DK-2800 Kgs. Lyngby, Denmark \\ ${ }^{b}$ Department of Chemistry, Faculty of Sciences, University of the Balearic Islands, \\ Carretera de Valldemossa km. 7.5, E-07122 Palma de Mallorca, Illes Balears, Spain
}

\begin{abstract}
Briefly looking back on the impact of flow injection analysis (FIA) as reflected in the rapid growth of publications in the scientific literature, and touching upon many of the novel and unique analytical chemical possibilities that FIA and its sequels, sequential injection analysis (SIA) and Lab-on-Valve (LOV), have offered, emphasis is placed on assays based on kinetic discrimination schemes, where, even subtle, differences in the reaction rates of occurring chemical reactions judiciously are exploited. A number of examples are given, covering homogeneous as well as heterogeneous conversions techniques, determinations of low levels of metals in complex matrices via suitable pre-treatment procedures, and soil fractionation schemes.
\end{abstract}

\section{Introduction}

Since the concept of Flow Injection Analysis (FIA) was first introduced in 1975 [1], it has had a profound impact on how modern analytical procedures are implemented. This is amply reflected in the many scientific publications that it has generated in all corners of the world: thus by the middle of 2006 more than 16.500 papers had been published to which should be added well over 20 monographs and numerous theses [2]. The reason for this momentum is clearly that FIA has allowed us to execute novel and unique procedures, which are difficult and, in many cases, not even feasible by the traditional batch procedures, the use of which literally for centuries have dominated the analytical scene. Or maybe more precisely expressed: generation upon generation of analytical chemists were taught, or rather indoctrinated, to believe that this was the only sensible way to perform chemical assays. With the advent of FIA, this conceptual restriction was exorcized, and as

${ }^{1}$ Corresponding author:

E-mail: ehh@kemi.dtu.dk; Tel.: +45 4525 2346; Fax: +45 45883136 
a result we have witnessed a surge in the development of new analytical approaches and applications. Yet, common for all them is that they are relying on the exploitation of the three cornerstones of FIA as already verbalized in the very first FIA-publication, namely: (i) injection, or insertion, of discrete, well-defined volume of sample solution into a flowing carrier stream (inert or itself a reagent, additional reagents being added subsequently); (ii) reproducible and precise timing of the manipulation that the injected sample zone is subjected to in the system, from the point of injection to the point of detection, that is, the so-called controlled, or rather controllable, dispersion; and (iii) the creation of a concentration gradient of the injected sample, providing a transient, but strictly reproducible readout of the recorded signal. The eventual readout, as monitored by a suitable detection device, is therefore always a result of two kinetic processes which occur simultaneously, namely the physical process of zone dispersion and the superimposed chemical processes resulting from reaction between analyte and reagent species.

As a result of growing environmental demands for reduced consumption of sample and reagent solutions, the first generation of FIA (or just FI, to emphasise that it is a conceptual approach, in addition to a means of performing analysis), which utilizes continuous pumping of carrier and reagent solutions, was in 1990 supplemented by the second generation, termed Sequential Injection Analysis (SIA - equally well often referred to merely as SI) [3,4]. Fully computer controlled and based on the use of a multi-position valve from the ports of which individual, precisely metered zones of sample and reagent(s) sequentially are aspirated by means of a syringe pump and stacked in a holding coil, and then subsequently, under dispersion within each other, are forwarded to a suitable detector, the SI-system implies not only a substantial savings in consumables, but inherently also in waste generation. And in 2000 we sat eyes on the appearance of the third generation of FIA, the so-called Lab-on-Valve (LOV), where even further downscaling was achieved and the concept of bead-injection (BI) involving bead renewal approaches was introduced, additionally offering new avenues for chemical assays [5-7]. And within the last couple of years SIchromatography (SIC) has emerged, permitting low-pressure separation procedures and analysis of multicomponent samples, especially facilitated via incorporation of commercially produced Chromolith columns (Merck), formed from a single piece of porous silica gel (monolith) $[8,9]$.

While much of the attention in using FI initially was set on the feasibility of achieving high sampling rates, as facilitated by exploiting transient rather than conventional steady-state signals, the focus was soon shifted to exploitation of the concentration gradient created, which, as a result of the axial and radial dispersion processes, in reality corresponds to an innumerable number of 
sequential liquid segments representing all concentrations from zero to the maximum of the FI peak readout, each of which potentially can be used for the analytical readout. This, in turn, gave rise to a number of gradient methods [10], among which especially should be highlighted the stopped-flow method, that has proven to be a very powerful tool in many contexts. Not the least for enzymatic procedures, both for assay of substrates and for determination of enzymatic activities, where the latter one traditionally has been very difficult to execute.

Later followed the introduction of methods relying on detection by bio- and chemiluminescence, where alpha and omega is the inherent and accurate timing of the FI-manifold, thus allowing to relate the maximum intensity of the generated transient light emission to the analyte concentration. These very sensitive analytical procedures, which prior to the introduction of FI virtually were non-existent, have blossomed to an extent that more than 1600 publications have been emerged in the scientific literature over the years, thus accounting for ca. $10 \%$ of all the published FI-papers. Essentially, these assays are all based on enzymatic conversion procedures, and these types of kinetic modus operandi are, in fact, some of the most frequently encountered ones. In the beginning primarily relying on the use of solubilised enzymes, but later predominantly via immobilised enzymes affixed on various supports and packed in column reactors, taking advantage of the fact that the costly enzymes, even though they are participating in the reactions with the substrate, are not consumed and thus can be reused. This again is reflected in the many publications, where the word "enzym*" is found in the title of more than 900 papers. And evidenced in the numerous reviews, among them the biannual reviews on kinetic methods which up to 2002 regularly were published in Analytical Chemistry.

Also intermediate/metastable constituents of specifically attractive analytical characteristics, in contrast to the ultimately formed products, have been utilised to serve for the analytical readout [11]. Yet, among the many exciting novel techniques we would especially like to emphasize the ones based on the so-called kinetic discriminating schemes, where, even subtle, differences in the reaction rates of occurring chemical reactions judiciously are exploited. Because, in our opinion, these conversion methods more than anything else demonstrate the unique capabilities of FI and its sequels to perform novel and original applications. And since it is impossible even to attempt to cover all aspects of FI, we have, for the very same reason, opted to devote the present communication to a closer survey of these schemes, as illustrated by some selected examples. The ones we have chosen do not, of course, pretend to be exhaustive, rather they have been picked subjectively to demonstrate different intriguing approaches. 


\section{Exploiting homogeneous conversion reactions}

\subsection{Determination of chlorate}

One of our favourite examples to demonstrate the essence of kinetic discrimination schemes is the determination of chlorate via a homogeneous conversion procedure. It has been picked, firstly because it is simple, yet elegant, and secondly because it is one of the first (if not the first) practical example presented in the literature [12]. Aimed at analysing chlorate in process liquor, the reaction scheme is as follows:

$$
\begin{aligned}
2 \mathrm{ClO}_{3}^{-}+10 \mathrm{Ti}^{3+}+12 \mathrm{H}^{+} & \longrightarrow 10 \mathrm{Ti}^{4+}+\mathrm{Cl}_{2}+6 \mathrm{H}_{2} \mathrm{O} \\
\mathrm{Cl}_{2}+\mathrm{LMB} & \longrightarrow \mathbf{M B} \\
\mathbf{M B}+\mathrm{Ti}^{3+} & \longrightarrow \mathrm{LMB}+\mathrm{Ti}^{4+}
\end{aligned}
$$

The assay is performed by injecting a sample of chlorate into an acidic carrier stream of titanium(III), which is subsequently merged with a second stream of leucomethylene blue (LMB). While the first two of these reactions are very fast, the reduction of the blue species MB by the third reaction is slow. Thus, taking advantage of the fact that the total residence time for the sample plug is very short within the FIA-manifold, the chlorate concentration can readily be quantified via the absorbance of the MB species generated by the second reaction, while the re-formation of LMB takes place after the sample plug has passed the detector and is directed to waste. Even if some MB should be reduced prior to entering into the detector, the precise and reproducible timing in the FIAsystem will ensure, that the fraction reduced always is precisely the same.

\subsection{Determination of phosphate and silicate}

The international ubiquitously used spectrophotometric standard methods for the determination of phosphate and silicate are both based on reaction with molybdate, leading to the formation of yellow heteropoly molybdoanions, which, for increased sensitivity of measurement, subsequently can be reduced to yield the well known intense molybdenum blue colour [13]. Citric, tartaric or oxalic acid is added in order to improve the selectivity of either reaction. Hence, it might appear paradoxical that the very same reactants and masking agents can be used for assaying each of the two components in the presence of each other. For the batch assay of phosphate and silicate according to the standard procedures, the secret lies in the sequence of adding the constituents [1416]. The explanation is to be found in the rates of the reactions taking place: 


$$
\begin{gathered}
\text { molybdophosphate }+ \text { tartrate } \stackrel{\text { fast }}{\rightleftarrows} \text { phosphate }+ \text { molybdotartrate } \\
\text { molybdosilicate }+ \text { tartrate } \stackrel{\text { slow }}{\rightleftarrows} \text { silicate }+ \text { molybdotartrate }
\end{gathered}
$$

Therefore, when tartaric acid is added last to a mixture of the two heteropoly anions, molybdophosphate is rapidly degraded, whereas molybdosilicate remains for sufficient time to be reduced to molybdenum blue and measured. When a composite reagent containing molybdate and tartaric acid is initially added to a mixture of phosphate and silicate, a small amount of molybdophosphate is formed in equilibrium with the other constituents. This is quickly reduced to molybdenum blue in the presence of a reducing agent, and more molybdophosphate is formed to restore the equilibrium. This continues until all the phosphorus is converted to molybdenum blue. The molybdosilicate is formed only slowly in the presence of tartrate, and does not interfere with the phosphate determination.

According to the standard method, the solutions should be left for a period of 10-30 minutes prior to measurement (primarily due to the relatively slow reduction of $\mathrm{Mo}(\mathrm{VI})$ to $\mathrm{Mo}(\mathrm{V})$ ). In the conventional continuous flow systems such long delay times were actually incorporated. Yet, considering the chemistries involved, they present themselves superbly for assaying the two analytes via the kinetic discrimination scheme. Therefore, it is not surprising that this approach already early was exploited in FI-manifolds [17] and later also in SI-systems [18,19].

In this context it should be added that the $\mathrm{pH}$ plays an important role in the kinetics of the assay. Thus, the yellow heteropoly molybdoanion complexes exist in two forms depending on $\mathrm{pH}$. The $\alpha-$ isomer is formed at $\mathrm{pH} 3.5-4.5$ and is a very stable once formed, whereas the $\beta$-isomer is formed rapidly in the $\mathrm{pH}$ range $0.8-2.5$, and is much more reactive. Hence, most analytical procedures are based on the formation of the $\beta$-isomers, although a variety of reaction conditions have been used reflecting the complex chemistry of the reactions [20]. This is also manifested when phosphate alone is the species of interest and the on-line formation of molybdosilicate is to be precluded in which case the acidity of the reaction medium is advantageously increased. Yet this should be weighted against the fact that the higher the acidity the lower the method's sensitivity becomes due to the slow development of the reaction for the molybdophosphate formation.

In the standard methods $\mathrm{Sn}(\mathrm{II})$ is recommended as reducing agent, while ascorbic acid already was suggested as a simple and effective alternative in the very first FI-papers published [1,21]. It was thus experimentally found that it was preferential for the determination of phosphate to premix 
the solutions of molybdate and ascorbic acid on-line before injecting the phosphate-containing sample, rather than injecting the sample into the molybdate stream and subsequently merging it with the ascorbic acid. Hence, seen in retrospect, these experiments actually verified (at that time, unknowingly) what was discussed above, with the added feature that beside acting as masking agent in the same manner as e.g. tartrate, ascorbic acid can also facilitate the reduction of Mo(VI) to $\mathrm{Mo}(\mathrm{V})$, as expressed in the following reaction sequences:

1 heptomolybdate + ascorbic acid + phosphate $\stackrel{\text { fast }}{\rightleftarrows}$ molybdenum blue

or

$2\left\{\begin{array}{r}\text { heptomolybdate }+ \text { phosphate } \stackrel{\text { fast }}{\rightleftarrows} \text { yellow } \text { heteropoly complex } \\ \text { yellow heteropolycomplex }+ \text { ascorbic acid } \stackrel{\text { sams molybdenum blue }}{\rightleftarrows} \text { mollo }\end{array}\right.$

That is, when a premixed mixture of molybdate and ascorbic is used (Eq. 1), the reactive intermediate of the reduced form of molybdate will be readily available and reactive with the injected phosphate sample, starting the generation of the molybdenum blue very fast (in the order of seconds). Whereas when the phosphate is injected into a molybdate stream the formation of the yellow product of molybdophosphate is observed which is not as reactive (Eq. 2), thus resulting in a slow reduction and hence generation of molybdenum blue (of the order of minutes). Grudpan et al. [22] exploited kinetic discrimination for determination of phosphate and silicate by means of stopped-flow measurements in an FI-system, and taking advantage of the fact that the reaction of phosphate-molybdenum-ascorbic acid is faster than that of silicate-molybdate-ascorbic acid they succeeded in resolving the individual signals and determine the two constituents.

\subsection{Hydride generation schemes}

Several elements (such as $\mathrm{As}, \mathrm{Sb}, \mathrm{Bi}, \mathrm{Se}, \mathrm{Te}$ and $\mathrm{Ge}$ ) can, by reaction with a strongly reducing agent, such as sodium tetrahydroborate, become chemically converted to their hydrides, according to the reaction shown below (Eq. 1). The gaseous hydrides can subsequently readily be separated from the sample matrix in a gas-liquid separator, and guided, via an auxiliary gas flow of nitrogen, to the heated quartz flow-through cell of an atomic absorption spectrometer (AAS) or the flame/plasma of an atomic fluorescence spectrometer (AFS). Here the hydrides are atomised by heating and excited by radiation, so that the elements of interest can be selectively quantified (Eq. 
2). Originally, the hydride generation technique was introduced as a batch procedure, but this involved several problems due to concurrently happening side reactions and interferences (Eqs 3-5):

Hydride generation/atomization:

$$
\begin{aligned}
& \mathrm{As}^{3+}, \mathrm{Sb}^{3+}, \mathrm{Sn}^{4+} \stackrel{\mathrm{BH}_{4}^{-}}{\longrightarrow} \mathrm{AsH}_{3}, \mathrm{SbH}_{3}, \mathrm{SnH}_{4} \\
& \mathrm{AsH}_{3}, \mathrm{SbH}_{3}, \mathrm{SnH}_{4} \stackrel{\Delta\left(900^{\circ} \mathrm{C}\right)}{\longrightarrow} \mathrm{As}, \mathrm{Sb}, \mathrm{Sn}+\mathrm{nH}_{2}
\end{aligned}
$$

Side reactions/interferences:

$$
\begin{aligned}
& \mathrm{BH}_{4}^{-}+3 \mathrm{HX}+\mathrm{H}^{+} \longrightarrow \mathrm{BX}_{3}+4 \mathrm{H}_{2} \\
& \left.\mathrm{Me}^{2+}(\mathrm{Ni}, \mathrm{Cu}, \mathrm{Co}) \stackrel{\mathrm{BH}_{4}^{-}}{\mathrm{Acid}(\mathrm{HX})} \mathrm{Me}^{0} \text { (slower }\right) \\
& \mathrm{AsH}_{3}, \mathrm{SbH}_{3}, \mathrm{SnH}_{4} \stackrel{\mathrm{Me}^{0}}{\longrightarrow} \mathrm{As}, \mathrm{Sb}, \mathrm{Sn}+\mathrm{nH}_{2}
\end{aligned}
$$

While the conversion of the analyte itself necessarily must take place in acidic medium, the tetrahydroborate itself can also react with acid and form hydrogen (Eq. 3), whereby the reagent is wasted for the hydride formation (although some generation of hydrogen actually is advantageous). Therefore, the tetrahydroborate must be prepared in a weakly alkaline medium and mixed with the sample and the acid precisely when it is required, and under very controlled conditions. A serious possibility for interference is the presence of free metals or metal boride precipitates, particularly of $\mathrm{Ni}, \mathrm{Cu}$ and $\mathrm{Co}$ [23]. If ionic species of these metal constituents are present in the sample, they become reduced by the tetrahydroborate, giving rise to the formation of colloidal free metals (Eq. 4) or metal borides, which have been shown to act as superb catalysts for degrading the hydrides before they can reach the measuring cell (Eq. 5). However, because of the dynamic conditions prevailing in FI, and because of the inherently short residence time of the sample within the system, these side reactions can to a large extent be eliminated or kinetically discriminated against at the expense of the main reaction. Again, if side reactions do occur, the precise timing of the FI-system ensures that they take place at exactly the same extent for all samples introduced [24,25]. A concrete example will readily illustrate this: In his work, Åström [26] found that it was totally impossible to determine minute quantities of $\mathrm{Bi}(\mathrm{III})(25 \mu \mathrm{g} / \mathrm{l})$ in the presence of $100 \mathrm{mg} / \mathrm{l} \mathrm{Cu}(\mathrm{II})$ in a batch system, because the hydride formed was degraded before it could reach the detector. However, when implementing the very same analytical procedure in a FI-system it was perfectly feasible. In fact, it yielded close to $100 \%$ response, that is, the interference due to $\mathrm{Cu}$ was practically eliminated. 


\section{Exploiting heterogeneous conversion reaction}

\subsection{Determination of low levels of metals in complex matrices}

In recent years a substantial part of our research activities have been focused on the determination of low concentrations of metal ions in complex matrices. In order to obtain the optimal sensitivity and selectivity, advantage has been taken of using atomic absorption or emission spectrometric detection methods such as FAAS, ETAAS, ICP-MS, ICP-AES and ICP-AFS. Yet, although these instruments are some of the most sensitive detection devices available, they are all, to some extent, prone to interferences (spectroscopic and/or non-spectroscopic), especially if the matrix contains high levels of salts. Therefore, it is frequently necessary to subject the sample to appropriate pretreatments, that is, to separate the analyte from potentially interfering matrix constituents, permitting at the same time to accomplish analyte preconcentration, which, for very low analyte concentrations, might be called for to bring the concentration within the dynamic working range of the detector

Such pretreatment procedures are advantageously implemented by FI- and SI-procedures, and lately also by the lab-on-valve concept (LOV) [27-30]. In our group we have used a number of online pretreatment procedures such as solvent extraction, solvent extraction/back-extraction, solidphase microcolumn extraction involving ion-exchange, chelation, or hydrophobic interactions, hydride generation, precipitation/coprecipitation, and sorption of neutral complexes in PTFE knotted reactors. Of particular interest are the assays based on solid-phase extractions (SPE), because they offer a great extent of versatility. Conventionally, SPE-procedures have been implemented by the use of permanent packed column reactors, yet in long term operation these reactors are prone to problems due to the following: (i) progressively tighter packing of the column material (e.g., beads or turnings) resulting in increase of back pressure; (ii) carry-over effects; (iii) variations in sorbent volume; (iv) malfunctions of the active entities, including loss of functional groups, the latter being a common problem for reagent-impregnated bead materials; and (v) surface deactivation due to irreversible interfering species. All these problems can be eliminated by adapting the concept of renewable surfaces, or, as it has been termed, bead injection (BI), that is, where the solid-phase material, if called for, can be renewed for each analytical cycle. This is readily feasible to implement in the LOV-approach, which thus, in this context constitutes to act as an ideal front-end for appropriate sample manipulation prior to the introduction of the analyte species into the atomic spectrometric detector used. 
In order to be operated in the micro SI-BI-LOV system ( $\mu$ SI-BI-LOV), some stringent requirements to the solid-phase materials employed must be fulfilled. Thus, they must be perfectly spherical (i.e., in the form of globe-shaped particles), uniform in size distribution (falling within a range of $40-150 \mu \mathrm{m}$ ), and possess a density close to that of water. Basically, there are three types of beads which are applicable, that is, either hydrophilic materials (having at their surface chelating or ion-exchange groups), or hydrophobic materials (typically covered with $\mathrm{C}_{18}$-surface entities), or materials which possess a combination of both characteristics. All these bead materials have been used in our research activities on metal determinations, the hydrophobic beads being particularly interesting, because they, via their ability to adsorb non-charged complex species, allow to design dedicated and selective chemistries by intelligent choice of the ligand used for the complex formation. While these procedures ostensibly appear to be straightforward, they are nevertheless often hampered due to kinetic problems. A few examples will illustrate this, as accompanied by appropriate solutions to the shortcomings.

One of our projects was aimed at determination of $\mathrm{Cr}(\mathrm{VI})$ via the well-known very selective reagent 1,5-diphenylcarbazide (DPC), using a hydrophobic bead material (poly(styrenedivinylbenzene) with pendant $\mathrm{C}_{18}$-moities) to capture the generated complex, and subsequently releasing it by an appropriate eluent for ensuing ETAAS determination of the retained $\mathrm{Cr}$ [30]. Based on initial experiments, where streams of $\mathrm{Cr}(\mathrm{VI})$ and DPC were merged within the LOV system, it became apparent that preciously little analyte became adsorbed on the beads. However, by pretreating the naked beads with DPC off-line, and then using these beads for capturing the $\mathrm{Cr}$ species, the procedure worked most successfully. The explanation for this behaviour is due to a combination of two facets: Firstly, the adhesion of DMG to the beads is a very slow process (it requires at least $30 \mathrm{~min}$ in an off-line fashion), and secondly the kinetics of the reaction of $\mathrm{Cr}(\mathrm{VI})$ and the DPC is actually rather complex, as illustrated by the following reaction sequence:

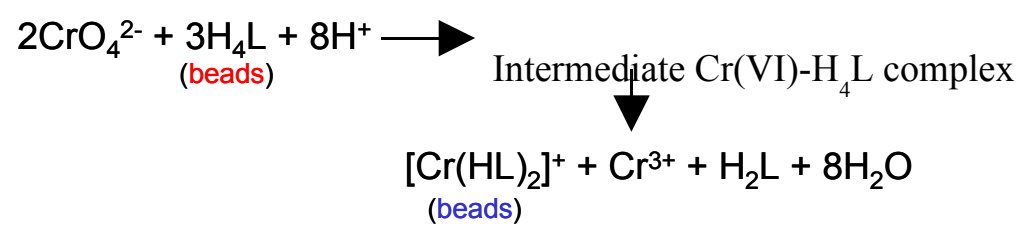

Thus, first a complex is formed between the $\mathrm{Cr}(\mathrm{VI})$ and the carbazide $\left(\mathrm{H}_{4} \mathrm{~L}\right)$ sitting on the beads, and then the $\mathrm{Cr}(\mathrm{VI})$ oxidises the carbazide to carbazone $\left(\mathrm{H}_{2} \mathrm{~L}\right)$, whereby (ideally) half of the generated $\mathrm{Cr}(\mathrm{III})$ is complexed by the immobilized carbazone and hence retained on the beads. 
Once retained, the complex can afterwards be eluted (we used methanol). Besides taking advantage of the fact that off-line pre-treatment procedure can be operated under optimal reaction conditions (e.g., $\mathrm{pH}$, time, temperature and reagent concentration), this approach entails two additional features: (1) Since the beads can be renewed for each analytical cycle, it does not matter how the metal ion is released from the beads, that is, whether it is eluted as a free metal ion while the ligand remains sorbed on the surfaces, or liberated as the complex itself, or as split up in $\mathrm{Cr}$ (III) ions and carbazone entities, or as a combination of the various possibilities (see Fig 1). And (2) the exact and reproducible timing inherent in the LOV-system will ensure that it is neither necessary to obtain the ideal $50 \%$ adhesion of $\mathrm{Cr}$ (III) on the beads, nor to achieve quantitative elution. In fact, as it turned out, we actually only obtained a $39 \%$ retention/elution efficiency, yet gained satisfactory results. Furthermore, as the sample solution is acidified on-line, any potential pre-reduction of $\mathrm{Cr}(\mathrm{VI})$ to $\mathrm{Cr}$ (III) that might occur in acidic media under the presence of potentially reducing species (such as organic matter) is depressed or eliminated.

This investigation actually gave us the impetus to propose the socalled 'universal approach' (Fig. 1) for pre-impregnation of ligands to bead material, implying that whatever ligand that can be adsorbed is applicable - irrespective of the kinetics involved - since the off-line pretreatment conditions readily can be executed under most favourable conditions. Furthermore, since the beads can be replaced for each cycle, the release of the sorbed complex species can equally well be performed under conditions which from an operational point of view are found to be optimal [30].

The second example deals with our recent work on determining Ni(II) by the traditionally used reaction with dimethylglyoxime (DMG) [31]. In this investigation we used a bead material, poly(divinylbenzene-co-N-vinylpyrrolidone, which possesses both hydrophilic and hydrophobic properties (ca. 50/50). Again, it appeared as a simple procedure, yet the merging of streams of $\mathrm{Ni}(\mathrm{II})$ and the DMG did not give rise to any signal, thus indicating that no preconcentration on the beads had taken place (Fig. 2). Further experiments revealed that the sparingly water-soluble $\mathrm{Ni}(\mathrm{DMG})_{2}$ chelate has to be generated prior to its exposure to the bead material in order to be retained. Thus, it was necessary to incorporate an externally attached mixing coil to the LOVsystem (Fig. 3), which, although operated in a nearly continuous back-and-forward mode, along with the liquid manipulations within the LOV system, allowed a delay time of ca. $70 \mathrm{~s}$ before the mixture containing the generated chelate was exposed to the beads. These measures proved to be sufficient for obtaining a retention efficiency of $69 \%$, allowing a preconcentration factor of 25 and a detection limit of $0.05 \mathrm{~g} \mu \mathrm{g} \mathrm{L}^{-1}$ at a reproducibility level of $5.6 \%$. 
Sample manipulations in the FIA-, SIA- and LOV-systems have thus not only proven to constitute effective approaches to vastly reduce, and even eliminate, the kinetic problems that one often encounters in batch assays, but to yield new and novel possibilities, which, although challenging, make these systems very promising for executing analytical assays. And besides testing the ingenuity of the analytical chemist - they are also the source of much exciting experimental work. Not to mention pleasure and satisfaction of having realised some novel and original research.

In the following section an example of precisely this combination will be presented.

\subsection{Immobilisation of proteins on solid supports}

Taking advantage of the unique possibilities of applying BI-LOV spectroscopy (BIS), Jarda Ruzicka and his group in Seattle have for a numbers of years successfully conducted novel research on numerous types of bioassays. Recently they published a very interesting paper which beautifully showed how the dynamic approach of BIS can be exploited for investigations into the kinetics of protein immobilisation onto solid supports (in casu, agarose beads), finding some very surprising and, indeed, most useful information [32].

When performing such immobilisations it is evident that the chemistry used is the key component for successful production of selective supports. Thus, it must create a stable bond between the bioligand and the solid support, while the support itself must remain inert in order to avoid non-selective adsorption. The physical and chemical stability of the link between the protein and the support should be such that leakage of the bioligand is minimised when used, not only during the capture, but also during repeated elution of the target molecules from a column or a biosensing surface. As the literature reveals, chemistries and protocols for immobilisation of proteins have gradually evolved from "home made" recipes, through trial and error, into industrial standards.

The immobilisation procedure itself is conventionally carried out using a batch procedure, and the general concept has hereto been that in order to ensure a sufficient degree of immobilisation the mixture should be allowed to react for several hours. Ruzicka and coworkers suggested, however, that by taking advantage of the BIS approach and periodically sampling minute amounts of the beads from a well-stirred microreactor, followed by spectroscopic interrogation of the loaded beads within the LOV configuration, it should be possible to monitor the reaction progress, which in turn would reveal the rate of conjugation, regardless of how long it would take for the solution/surface 
chemistries to reach equilibrium (Fig. 4). Besides, such automated monitoring of the immobilisation chemistries, carried out in a well-defined, stirred reaction mixture, should furthermore allow reliable exploration and efficient optimization of the reaction conditions, such as $\mathrm{pH}$, buffer composition, and ligand concentration.

The results of their investigations using the BIS approach resulted in some very surprising discoveries. Thus, they found - in contrast to established protocols, accepted for a number of years - that the rate and amount of immobilised protein depends on the $\mathrm{pH}$ and on the protein $\mathrm{pI}$ (isoelectric p-value). Furthermore, with some proteins, such as lysozyme, cytochrome $\mathrm{C}$ and human $\mathrm{IgG}$, immobilisation equilibrium was actually reached in alkaline solutions within 10 minutes rather than on a scale of several hours. While literature data suggest that almost a $90 \%$ immobilisation yield can be achieved at $\mathrm{pH} 4-7$, the response curves obtained by BIS monitoring showed that below pH 7 almost no protein was being immobilized, and for proteins with $\mathrm{pI}<5$ alkaline solutions had to be used. Used cleverly, the BIS thus offered some most useful data and experimental insight into the kinetics of the reactions involved, allowing the authors most reflectively, yet also very appropriately, to add in their conclusion "One can only speculate how many unsuccessful attempts to immobilise proteins on agarose or other supports, including biosensing surfaces, were due to the use of media with too low $\mathrm{pH}$ ".

\subsection{Fractionation studies of solid materials.}

As an extension of studies for developing of analytical assays for trace metal determinations, this group has in the last few years conducted investigations into determining metals in solid materials (e.g., soils and sediments) via fractionation schemes. In such assays the solid sample is sequentially subjected to various leaching solutions which, via their composition, provide information of the potential availability of the metal species present in the sample. Thus, by using operatively defined and internationally accepted leaching agents of increasing aggressivity, one may distinguish between fractions such as "the exchangeable", "the acid soluble", "the reducible" and "the oxidisable" constituents of the sample. The rationale for conducting such studies is to provide information about the mobility of the metals present, and thus their potential toxicity, that is, their effects as anthropogenic agents.

Sequential extraction techniques are traditionally conducted in a batch end-over-end fashion, which is rather laborious, tedious, time consuming, and it is subjected to several potential errors including risks of contamination due to sample manipulation and underestimation of given fractions 
due to re-adsorption phenomena. Although naturally occurring processes inherently always are dynamic, batchwise extraction is nevertheless routinely performed with particular leaching agents for extended periods of time to ensure the establishment of steady-state conditions between the solid and liquid phases. Hence, no insight into the leaching kinetics can be obtained.Therefore, it is preferable to effect such schemes in a continuous-flow fashion, not the least because this will also more realistically mimic how the leaching processes are occurring in natura - and at the same time yield a more detailed information.

As a consequence, we have addressed this problem by designing and developing an entirely novel flow-though microcolumn extraction unit suitable for incorporation in an SI system allowing on-line operation [33-36]. Taking advantage of the fully computerised facilities offered by SI, the automated sequence comprises the consecutive aspiration of the individual extractants from different external ports of the valve, which, via flow reversal, sequentially are exposed to the solid sample as contained in the microcartridge attached externally to one of the port positions. Furthermore, it is feasible to exploit programmable uni-, bi-, or multi-bi-directional flow or even stopped-flow [34]. And coupled to an on-line detector, it can, via the very small extractant volumes delivered to the detector (of the order of 100-300 $\mu$ ), yield a very high concentration-time resolution [35] and thus a very detailed insight into extraction behaviour [37] as reflected in the recorded extractograms (Fig. 5).

A crucial issue when making these extractions is readsorption, that is, whether metals once released by an extracting agent can become trapped by the remaining solid material or freshly created surfaces, in which case the metal concentration will be underestimated and the concentration by the ensuing extractant will be overestimated. Inherently, such readsorption phenomena are most likely to take place if the extractant is exposed to the solid material for an extended period of time. This is therefore most likely to occur under batch conditions, while in the dynamic approach, where the leachant is continuously renewed, the metal redistribution should be kinetically reduced to a large extent [37]. As the experiments revealed, the SI-approach actually eliminated the readsorption completely.

Recently we have also used the dynamic SI-approach for determining macro-nutrients, more specifically orthophosphate [38,39]. Using $\mathrm{NH}_{4} \mathrm{Cl}, \mathrm{NaOH}$ and $\mathrm{HCl}$ as leaching agents, it is important that the extractants only allow the accurate determination of the various orthophosphate pools, and at the same time ensure that the hydrolysis of extracted organic phosphorus and condensed inorganic phosphates within the time frame of the assay is kept at minimum. Here again, 
we can play on the kinetics, because while such hydrolysis readily might occur in batch measurements, the on-line approach leads to a substantial shortening of the assay protocol, thus minimizing the potential decomposition of hydrolyzable phosphorus compounds. Each microvolume of extract leaving the microcolumn can subsequently readily be treated on-line with molybdenum blue (MB) reagents, while, in the batchwise method, the released organic species remain in intimate contact with the extracting reagent for extended periods of time (of the order of hours). Therefore, the flow-through FI-fractionation analyzer with MB detection should be regarded as a unique tool for accurate monitoring of free, readily mobilisable orthophosphate in environmental solid substrates, even though organic phosphorus might be leached with the extracting reagents [39].

\section{Conclusion}

As demonstrated, the exploitation of kinetic discrimination schemes in FI/SI/LOV systems has provided an extra degree of freedom in executing chemical assays, thus allowing novel and unique applications. This versatility is primarily due to the capability of both propelling and aspirating liquid streams at a user-defined flow rate - and, if necessary, also solid materials such as beads and, equally important, the possibility of performing stopped-flow, all unit operations being feasible at will. These characteristics are imperative in performing on-line analytical measurements if we wish not merely to rely on the thermodynamics of the reactions involved, but also to take advantage of their kinetics. Therefore, the FI/SI/LOV systems promise to entail much higher potentials than their $\mu$-TAS or nano-counterparts, where especially the liquid drivers are the bottleneck, not to mention systems relying on electrophoretic solution transport. Besides, due to the small channel dimensions in these miniaturised systems, the solutions used must necessarily be ultrapure, because the presence of even minute amounts of solid particles will cause clogging problems. From the literature it is furthermore apparent that research with these micro-/nano-devices, due to their inherent very short residence times, is limited to very fast chemistries. This is not only a severe restriction seen from an analytical chemical point of view, but at the same time it sets boundaries as to developing and using interesting, intelligent and, not to forget, entertaining chemistries.

For the very same reasons, we are embarked on taking advantage of the automated FI/SI/LOV systems in our future research activities.

\section{Acknowledgement}


The authors wish to extend their thanks to the mechanical workshop at the Department of Chemistry, Technical University of Denmark, for their invaluable assistance in producing a number of crucial components. Manuel Miró is indebted to the Spanish Ministry of Education and Science for financial support through the Ramón y Cajal research program. 


\section{References}

[1] J. Ruzicka, E.H. Hansen, Anal. Chim. Acta 78 (1975) 145.

[2] E.H. Hansen, Flow Injection Bibliography; http://www.flowinjection.com/; 2006.

[3] J. Ruzicka, G.D. Marshall, Anal. Chim. Acta 237 (1990) 329.

[4] C.E. Lenehan; N.W. Barnett; S.W. Lewis Analyst 127 (2002) 997.

[5] J. Ruzicka, Analyst 125 (2000) 1053.

[6] A.D. Carroll, L. Scampavia, J. Ruzicka, Analyst 127 (2002) 1228.

[7] Y. Gutzman, A.D. Carroll, J. Ruzicka, Analyst 131 (2006) 809.

[8] D. Satinsky, P. Solich, P. Chocholous, R. Karlicek, Anal. Chim. Acta 499 (2003) 205.

[9] J. Klimundova, D. Satinsky, H. Sklenarova, P. Solich, Talanta 69 (2006) 730.

[10] E.H. Hansen, Fresenius Z. Anal. Chem. 329 (1988) 656.

[11] J. Ruzicka, E.H. Hansen, Flow Injection Analysis, 2nd edn, Wiley, New York, 1988.

[12] H. Matschiner, H. H. Ruettinger, P. Sivers, U. Mann, German (East) Pat. No. 216543, 1984.

[13] American Public Health Association, American Water Works Association, Water Pollution Control Federation, Standard Methods for Examination of Water and Wastewater:

Determination of P and Si, Washington DC, 20th edn., 1998, Ch.4.

[14] W.J. Williams, Handbook of Anion Determination, Butterworths, London, 1979, p. 467.

[15] R.A. Chalmers, A. G. Sinclair, Anal. Chim. Acta 230 (1966) 412.

[16] E.H. Hansen, Anal. Chim. Acta 261 (1992) 125.

[17] R. Kuroda, I. Ida, K. Ogama, Michrochim. Acta Part I (1984) 377.

[18] C.X. Galhardo, J.C. Masini, Anal. Chim. Acta 417 (2000) 191.

[19] S. M. Sultan, M. U. Legemah, J. Flow Inject. Anal. 22 (2005) 25.

[20] K. Robards, I.D. McKelvie, R.L. Benson, P.J. Worsfold, N.J. Blundell, H. Casey, Anal. Chim. Acta 287 (1994) 147.

[21] J. Ruzicka, J.W.B. Stewart, Anal. Chim. Acta 79 (1975) 79.

[22] K. Grudpan, P. Ampan, Y. Udnan, S. Jayasvati, S. Lapanantnoppakhun, J. Jakmunee, G.D. Christian, J. Ruzicka, Talanta, 58 (2002) 1319.

[23] S.Tesfalidet, K. Irgum, Anal. Chem. 1989, 61, 2079.

[24] J. Ruzicka, E.H. Hansen, Flow Injection Analysis. 2nd edn., Wiley, New York, 1988, Ch. 2, p. $15-85$.

[25] Z.-L. Fang, Flow Injection Separation and Preconcentration. VCH, Weinheim, 1993, Ch. 5, p. 121-156. 
[26] O. Åström, Anal. Chem. 54 (1982) 190-193.

[27]. E.H. Hansen and J.-H. Wang, Anal. Lett. 37 (2004) 345.

[28]. J.-H. Wang and E. H. Hansen, Trends Anal. Chem. 24 (2005) 1.

[20]. E.H. Hansen, M. Miró, X.-B. Long and R. Petersen, Anal. Lett. 39 (2006) 1243.

[30]. X.-B Long, M. Miró and E. H. Hansen, Anal. Chem. 77 (2005) 6032.

[31]. X.-B. Long, M. Miró, R. Jensen and E. H. Hansen, Anal. Bioanal. Chem. (in press).

[32] J. Ruzicka, A.D. Carroll, I. Lahdesmaki, Analyst 131 (2006) 799.

[33] R. Chomchoei, E.H. Hansen and J. Shiowatana, Anal. Chim. Acta 526 (2004) 177.

[34] R. Chomchoei, M. Miró, E.H. Hansen and J. Shiowatana, Anal. Chim. Acta 536 (2005) 183.

[35] R. Chomchoei, M. Miró, E.H. Hansen and J. Shiowatana, Anal. Chem. 77 (2005) 2720.

[36] M. Miró and E.H. Hansen, Anal. Bioanal. Chem.., 382 (2005) 878.

[37] M. Miró, E.H. Hansen, R. Chomchoei, W. Frenzel, Trends Anal. Chem., 24 (2005) 759.

[38] J. Buanuam, M. Miró, E.H. Hansen and J. Shiowatana, Anal. Chim. Acta 570 (2006) 224.

[39] J. Buanuam, M. Miró, E.H. Hansen, J. Shiowatana, J.M. Estela and V. Cerdà, Talanta (subm.). 


\section{Figure Legends}

Fig. 1. The concept of the universal approach, where the hydrophobic beads initially are preimpregnated off-line with the selected ligand, advantage being taken of operating under optimal reaction conditions to affix the ligand. These pretreated beads are then used for on-line retention of the analyte metal species, the elution and subsequent determination of the metal being unaffected of the mechanisms involved in the liberation of the retained chelate, because the beads are renewed for each sample cycle.

Fig. 2. Reaction between $\mathrm{Ni}(\mathrm{II})$ and dimethylglyoxime (DMG) and retention of the generated complex on a bead material with both hydrophilic and hydrophobic surface characteristics in the ratio 1:1. If the analyte and the reactant are added on-line in contact with the bead material, no adsorption of complex takes place. Thus, it is necessary to implement a delay time in the LOVsetup, allowing first the generation of the complex and subsequently its adsorption to the bead material. The actual $\mu$ SI-LOV-ETAAS system is shown in Fig. 3.

Fig. 3. Schematic diagram of the $\mu$ SI-LOV-ETAAS system for on-line determination of Ni(II) via precipitation with DMG and preconcentration on poly(divinylbenzene-co-N-vinylpyrrolidone) beads. Carrier, $0.2 \mathrm{~mol} \mathrm{~L}^{-1}$ ammonium citrate buffer ( $\mathrm{pH} 9.0$ ); DMG, 1.2\% (w/v) dimethylglyoxime in ethanol; Eluent, methanol; SP1 and SP2, Syringe pumps 1 and 2; $\mathrm{C}_{1}$ and $\mathrm{C}_{2}$, LOV micro-column positions; HC, Holding coil; RC, reaction coil; CC, Central communication channel; PP, Peristaltic pump, ETAAS, electrothermal atomic absorption spectrometer. In order to obtain sufficient delay time for the generation of the $\mathrm{Ni}(\mathrm{DMG})_{2}$ chelate prior to adsorption on the beads, the sample solution, as pre-aspirated into $\mathrm{HC}$, is initially mixed with DMG and then passed into an externally attached reaction coil, RC. The mixture is then, without any stop period, aspirated back into HC and finally passed to $\mathrm{C}_{2}$. Adapted from ref. 31 by permission of Springer Verlag.

Fig. 4. $\mu$ SI-system configured for bead injection spectroscopy (BIS). A. System setup: the hollow PEEK tubing "plug a" focuses the carrier stream into the center of the packed beads while "plug b" helps to retain the beads within the optical path. B. Flow cell configuration. C. Details of flow cell construction: (a) the optical fibers hold plug " $a$ " in place and (b) the Teflon tubing in the waste channel is cut at an angle to hold plug " $b$ " in place and to prevent clogging. Reproduced from ref. 32 by permission of the Royal Society of Chemistry. 
Fig. 5. Extractograms for the acid-soluble fraction of orthophosphate from SRM 2711 as obtained by using a flow-through microcolumn assembly and employing either ( $(\boldsymbol{\bullet})$ off-line and ( $\bullet$ ) on-line spectrophotometric detection. The subfraction volumes were $5 \mathrm{ml}$ and $250 \mu \mathrm{l}$, respectively. Reproduced from ref. 39 by permission of Elsevier Science Publishers. 


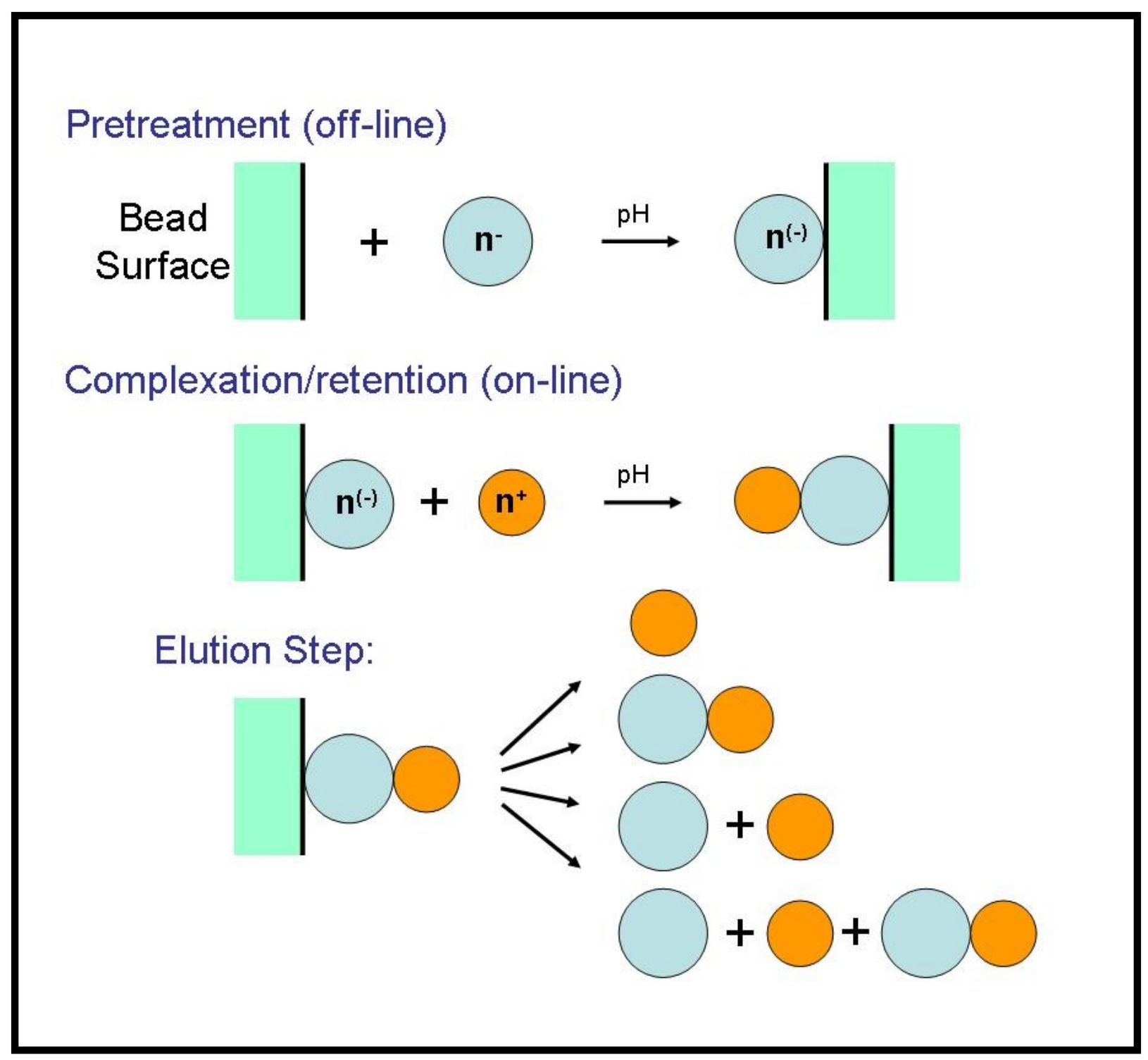

Fig. 1 
If solutions of $\mathrm{Ni}(\mathrm{II})$ and DMG are mixed on-line:

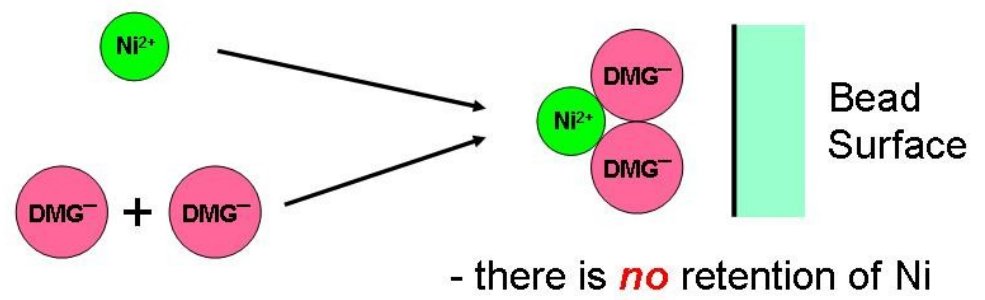

Due to the kinetics involved, the complex must be generated prior to exposure to the beads:

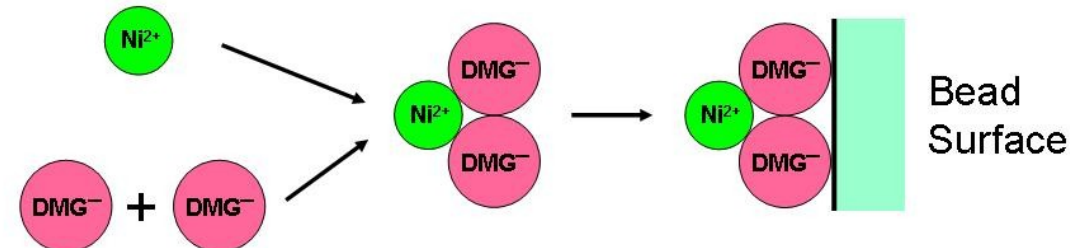

Fig. 2 


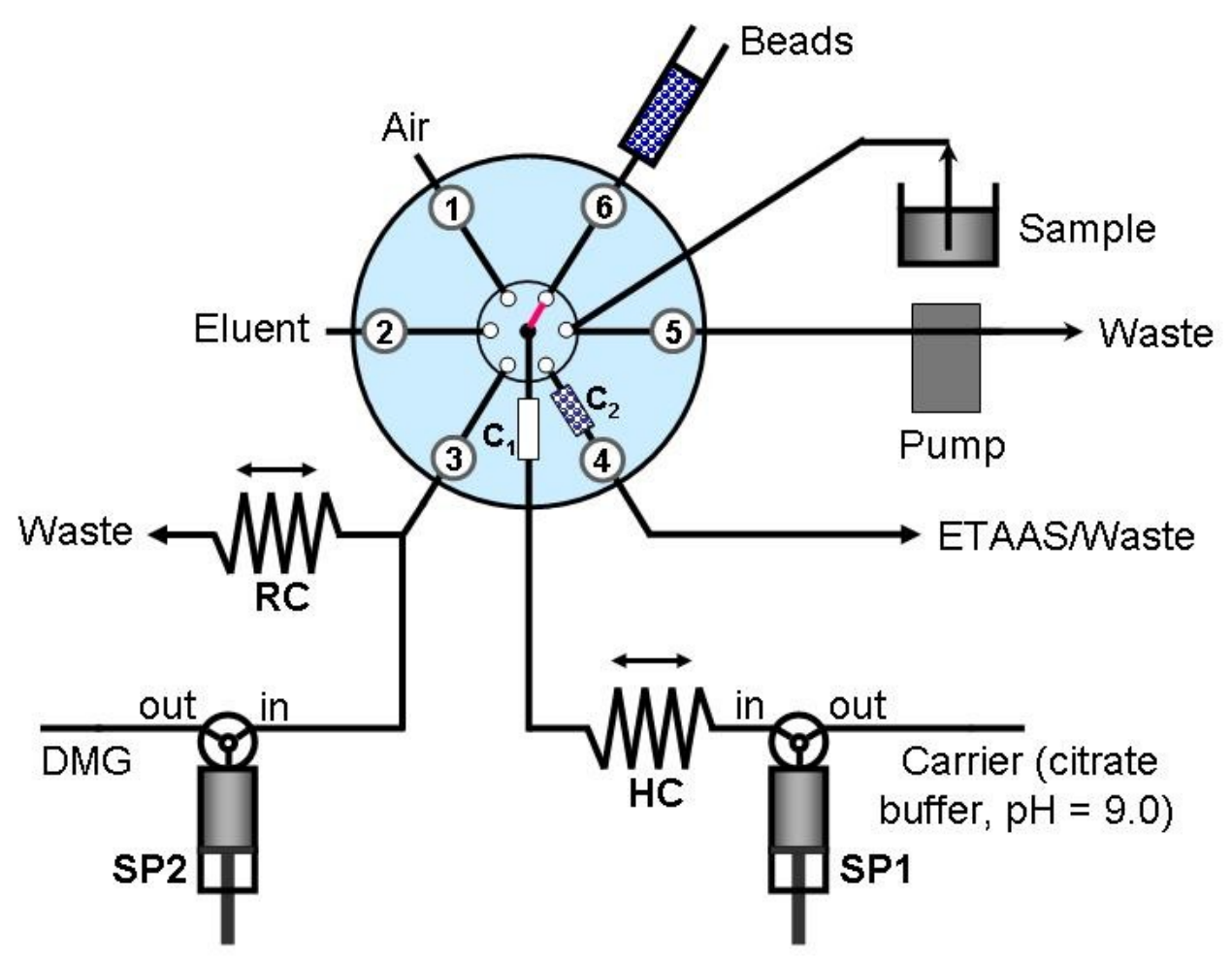

Fig. 3 


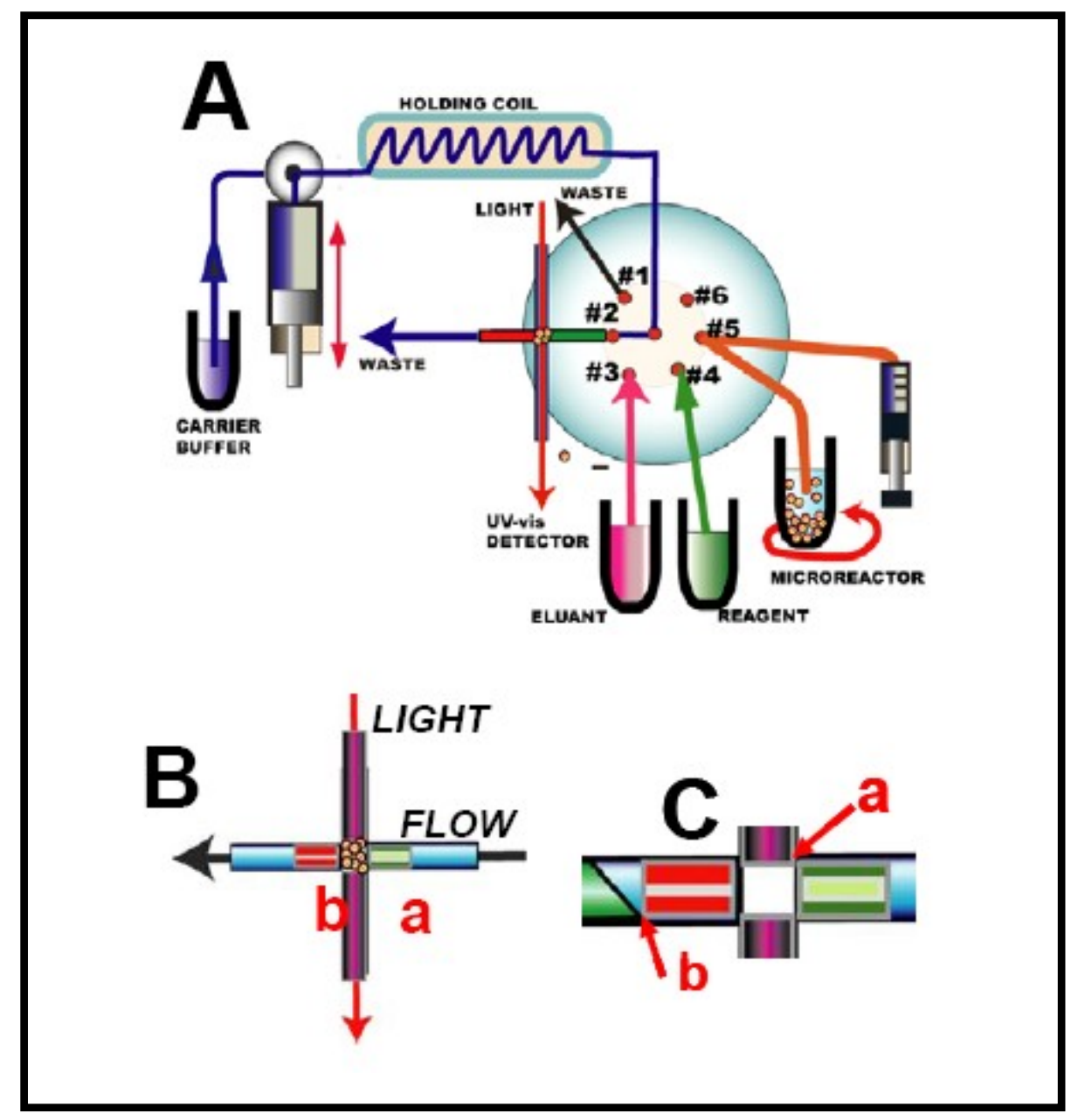

Fig. 4 


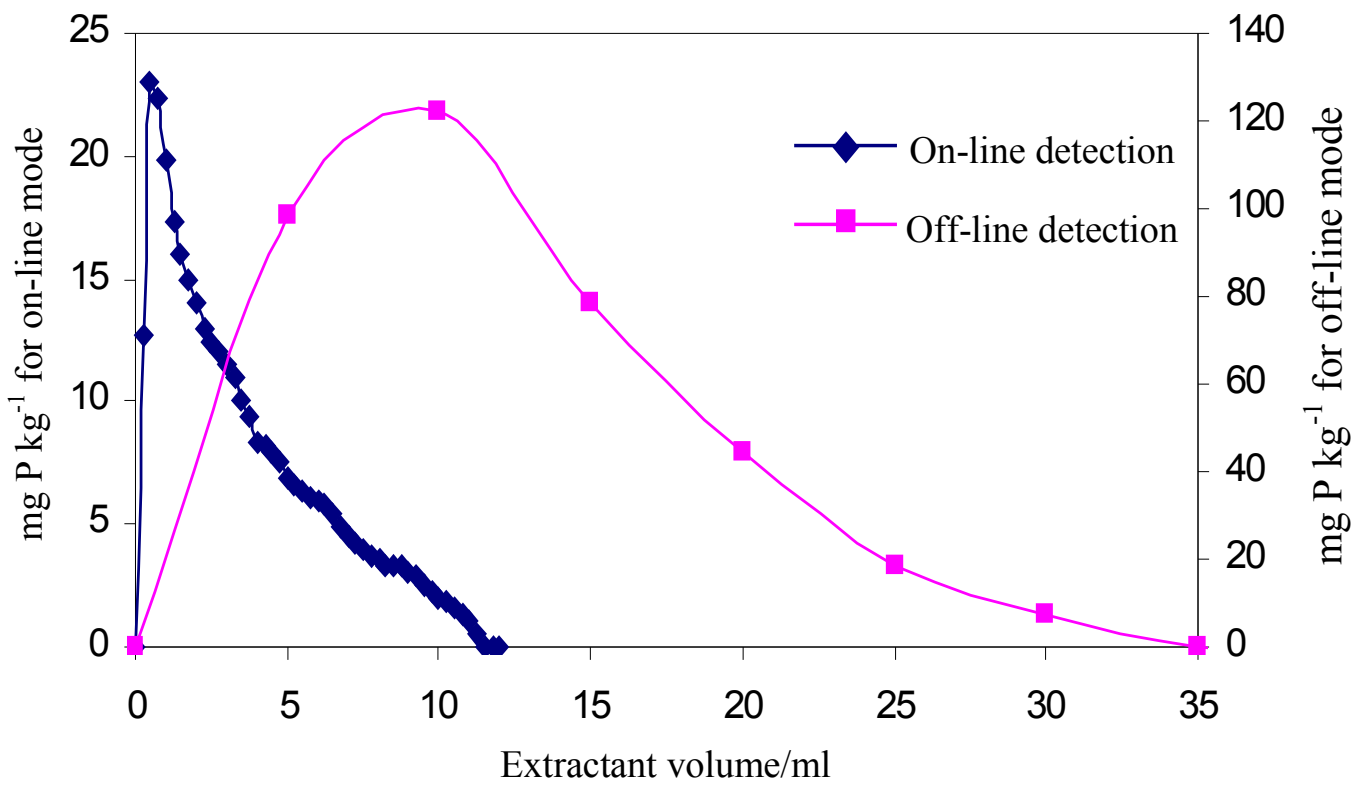

Fig.5 